\title{
Biological Aspect of Dates Supplementation in Lactating
}

\section{Rats' Diet}

\author{
Nahla A El-bostany*, Fathia M Soliman*; Maha Mahmoud*, Ali I S Ahmed*
}

\begin{abstract}
In the current study on lactation in rats' dates (D) ratio in diet was examined in supporting this physiological case. Dates were used both at 25 or $50 \%$ with another food sources such as skimmed milk (SM) at 10 or $30 \%$ plus a source for $\mathrm{CHO}$ such as sweet potatoes (SP) at a variable ratio of 10,25 , or $35 \%$. A special herbal mixture of extract as additives (AD) was added to all groups except the NC. The NC control composition was $46 \%$ both SM and corn starch (S), wherein the ratio of proteins in diet, almost $17.5 \%$, was corrected using 10 or $20 \%$ of a plant-rich protein antioxidant semimodified food (AC). Date has been found to be preferable up to $25 \%$ in a special combination as lactating diet. The food component of the best diet under the condition of this experiment, i.e., the diet met the most healthy both mothers and infants, were the skimmed milk (SM) of $10 \%$ plus sweet potatoes (SP) at a high ratio of $25 \%$. It seems that little higher ratio of proteins, i.e., $18 \%$, most of which are plants with plenty amount of vegetable $\mathrm{CHO}$ and less than $10 \%$ fat in oily form with an adequate amount of minerals and vitamins, particularly found in D and SP, are important food toleration for those rats. This is formally seen in pregnancy. Fortunately, the diet of choice according to the above mentioned role gave also the optimal BW measurement. However, rapid weight loss through diet or medication should not be attempted during lactation. Under the light of blood cell counts, blood analysis and both liver and kidney histopathology, the special extract used seemed to be important as source for some minor elements. The conjugation of those micronutreints and the macronutreints provided by the food system mentioned above has strongly supported that sort physiological nutritional requirements. Lactation is a special physiological case with a specific metabolic hormonal balance. This is an adapted hormonal shift (HS) follows this particular physiological status. It is such a hormonal program that strongly connected to feeding category. The present data show an alternative food combination that support mother health based on biological evaluation used, i.e., liver function and histology was group 4 of 25 D, 10 AC, 30 SM, and 25 SP. More investigation is needed to observe the role of the food additives used in the presence of main nutrients on this profounded kind of hormonal status.
\end{abstract}

\section{INTRODUCTION}

The questions were encountered health. To investigate the effects of concerning the nutritional status of lactating maternal nutrition on the milk, the infant, women and links between maternal and the mother herself, much more nutrition and both infant and maternal information is needed. Among those, the *Special Food \& Nutr, Food Technology Research Institute, Agric. Res. Center, Giza. Egypt 
interactions between milk production, infant demand, and maternal nutrient intake and stores, as well as on the transfer of nutrients from mother to milk and the factors regulating this process.

The following are some key elements of dietary advice for lactating women according to some specialized scientific organization: women who are breastfeeding do not need to eat any special diet (Bayol, 2007(1)). They should eat a regular healthy diet. Eat a variety of healthy foods from the food pyramid. Include three servings from the milk group, two servings from the fruit group and three servings from the vegetable group daily. A woman who is breastfeeding may be more hungry than usual and she should listen to her body and add additional servings from the fruit, vegetable and bread and cereal groups (Bayol, 2007)(1). Water, milk and fruit juices are excellent choices for the new mother. Minimize the amount of empty calorie foods consumed, so that there is more appetite for nutrient-rich foods. No one food or food group should be eliminated from the diet unless a specific infant has a very clear reaction to a specific food. Rapid weight loss through diet or medication should not be attempted during lactation (Bayol, 2007) $)^{(1)}$.

In brief, healthy and balanced diet is needed, mothers who eat junk food during pregnancy and breastfeeding may be putting their children at risk of overeating and developing obesity according to a study funded by the Welcome Trust and carried out at the Royal Veterinary College, London. The research suggests that pregnant and breastfeeding women should not indulge in fatty, sugary and salty foods under the misguided assumption that they are "eating for two". (Bayol, 2007)(1)

The junk food such as doughnuts, muffins, biscuits, crisps, and sweets during pregnancy and lactation gave birth to offspring which overate and had a preference for junk foods rich in fat, sugar 
and salt when compared to the offspring of rats given regular feed. The research team behind the study believes the findings have implications for humans. (Bayol, 2007)(1). The study has shown that eating large quantities of junk food when pregnant and breastfeeding could impair the normal control of appetite and promote an exacerbated taste for junk food in offspring. This could send offspring on the road to obesity and make the task of teaching healthy eating habits in children even more challenging. (Bayol, 2007) $)^{(1)}$

Controlling appetite is complex, involving hormones, which signal to the brain to regulate energy balance, hunger, and satiety or feeling of fullness. However, feeding is not only a matter of regulating energy balance; it is also a pleasurable experience that involves reward centers in the brain, such that the combination of pleasure with feeding may occasionally override the normal regulation of satiety. Previous research has shown that junk foods rich in fat and sugar inhibit the satiety signals while promoting hunger and stimulating the reward centers, (Bayol, 2007)(1). Exposure to a maternal junk food diet during their fetal and suckling life might help explain why some individuals might find it harder than others to control their junk food intake even when given access to healthier foods later in life. Mothers need to be made aware of the risks associated with a poor diet (Bayol, 2007)(1), that more needs to be done to raise awareness in pregnant and breastfeeding women as well. However, indicators are needed both of 1 - risks of adverse outcomes related to the mother's dietary intake and 2- the potential of the mother or her nursing infant to benefit from interventions designed to improve their nutritional status or health.

Biologically, Cripps and Williams (1975)(2) studied the food consumption, live weight, anatomical measurements of the gut organs, and the absorptive capacity of the small intestine for L-leucine and $D(+)$ - 
glucose that made on virgin (control), pregnant, and lactating albino rats. They found that food intake increased by approximately $60 \%$ during pregnancy and a further $250 \%$ during lactation. Organ anatomical shape may change with pregnancy and lactation. Pregnancy did not markedly influence the gross anatomy of the gastrointestinal tract. There was evidence for increased villus height and percentage water in the small intestine and for increased length of the colon during pregnancy. During lactation, the alimentary canal progressively increased in weight and size (Cripps and Williams, 1975(2)).

All anatomical measurements, except the length of the small intestine, completely regressed to control values within $20 \mathrm{~d}$ of weaning. The increased intestinal length had not completely regressed by day 30 post-weaning (Cripps and Williams, 1975)(2). Moreover, no significant change was observed in absolute absorption of glucose or leucine during pregnancy.
Absolute absorption of leucine and of glucose was increased during lactation. Greatest absorption occurred on the 10th day of lactation (Cripps and Williams, 1975)(2).

Neuropeptide $Y$ is the most powerful appetite stimulant known, and rates of synthesis and release in the hypothalamus correlate closely with nutritional status (Wilding, et al 2002)(3). Hypothalamic NPY mRNA was unchanged in pregnancy, moderately increased after 5 days lactation $(130 \pm 6.2 \%$ of control, $\quad \mathrm{P}<0.01)$ and increased further at 14 days lactation. Increases in hypothalamic NPY synthesis may be partly responsible for the increase in food intake seen in lactation (Wilding, et al 2002)(3).

Historically, more than two decades of research have established that breast milk is perfectly suited to nourish infants and protect them from illness. Breast-fed infants have lower rates of hospital admissions, ear infections, diarrhea, 
rashes, allergies, and other medical problems than bottle-fed babies (Williams, 1995(4). Breast-fed babies have fewer illnesses because human milk transfers to the infant a mother's antibodies to disease. About 80 percent of the cells in breast milk are macrophages, cells that kill bacteria, fungi, and viruses. Breast-fed babies are protected, in varying degrees, from a number of illnesses, including pneumonia, botulism, bronchitis, staphylococcal infections, influenza, ear infections, and German measles. Furthermore, mothers produce antibodies to whatever disease is present in their environment, making their milk custom-designed to fight the diseases their babies are exposed to as well.

Actually, there are 4,000 species of mammals, and they all make different milk. Human milk is made for human infants and it meets all their specific nutrient needs. The academy recommends that babies be breast-fed for six to 12 months. There aren't any rules about when to stop breast- feeding. A baby needs breast milk for the first year of life, and then as long as desired after that (Williams, 1995(4)).

In fact, the primary benefit of breast milk is that nutritional human milk contains just the right amount of fatty acids, lactose, water, and amino acids for human digestion, brain development, and growth. Cow's milk contains a different type of protein than breast milk. This is good for calves, but human infants can have difficulty digesting it. Bottle-fed infants tend to be fatter than breast-fed infants are, but not necessarily healthier. A breast-fed baby's digestive tract contains large amounts of Lactobacillus bifidus, beneficial bacteria that prevent the growth of harmful organisms. Human milk straight from the breast is always sterile, and never contaminated by polluted water or dirty bottles, which can also lead to diarrhea in the infant. Human milk contains at least 100 ingredients not found in formula. No babies are allergic to their mother's milk, 
although they may have a reaction to something the mother eats. If she eliminates it from her diet, the problem resolves itself.

Physiologically, during pregnancy body weight, organ, and particularly adiposity, increase due to hyperphagia rather than decreased energy metabolism. These physiological adaptations provide the growing fetus(es) with nutrition and prepare the mother for the metabolically demanding lactation period following birth. Mechanisms underlying the hyperphagia is still poorly understood (Douglas, et al, 2007)(5). However, cholecystokinin (CCK) secreted from the duodenum during feeding has been shown to elicit satiety and stimulate growth of the pancreas in addition to affecting gastrointestinal function. In previous experiments, hyperphagic Zucker obese rats were less sensitive to the effects of CCK on satiety and had a smaller pancreas than normalweight rats. In an experiments with hyperphagic lactating Zucker rats, the food intake response to exogenously administered CCK and the size and composition of the pancreas were measured (McLaughlin, et al, 1983(6)). Food intakes after a 2-h fast were not decreased by 4.0 or 8.0 micrograms $/ \mathrm{kg}$ CCK-8 during wk 1,2 , or 3 of lactation. However, in the same rats 2 wk after pups were weaned, 4.0 and 8.0 micrograms $/ \mathrm{kg}$ CCK-8 decreased food intake 32\% (2.1 +/0.4 vs. $3.1+/-0.3 \mathrm{~g}$, paired $t=2.33, P$ less than 0.03$)$ and $52 \%(1.5+/-0.2$ vs. $3.1+/-$ $0.5 \mathrm{~g}$, paired $\mathrm{t}=3.48$, $\mathrm{P}$ less than 0.006 ) On day 18 of lactation, pancreas weight was increased $41 \%$ (1.38+/- 0.05 vs. 0.98 $+/-0.02 \mathrm{~g}$, paired $\mathrm{t}=2.68, \mathrm{P}$ less than 0.02) and contents of DNA, RNA, and protein were increased 57,57 , and $73 \%$, respectively (McLaughlin, et al, 1983)(6). Thus, hyperphagia in lactating female rats was associated with: 1- decreased sensitivity to the satiety effect of CCK similar to that in hyperphagic obese rats 
and 2- hypertrophy of the pancreas in contrast to decreased pancreas size in obese rats (McLaughlin, et al, 1983)(6).

However, several adaptations in hypothalamic neuroendocrine systems may converge to increase ingestive behavior. Oxytocin is one of the anorectic hypothalamic neuropeptides. Oxytocin neurons, both centrally projecting parvocellular oxytocin neurons and central dendritic release of oxytocin from magnocellular neurons, may play a key role in regulating energy intake (Douglas, et al, 2007)(5).

Research is needed to develop indicators of nutritional status during lactation, to identify groups at risk of nutritional problems, and to determine effects of maternal nutritional status on various measures of lactation performance such as the volume and composition of milk, the duration of lactation, and infant growth and health .

Therefore, special diet or dietary supporting semimodified food is proposed here to meet this particular biological hormonal balance and the specific metabolic situation associated with lactation.

\section{MATERIAL AND METHODS}

Material: The food materials: Semisolid dates, powdered sweet potatoes and skimmed milk as well as honey, apple, and spices used in preparation of special additives (AD) were obtained from local market in Cairo. The ACE is an antioxidant plant protein formula first produced by Ahmed et al, 2005(7)).

Rations and Animals: Thirty adult female albino rats of western strains were housed in the Ophthalmology Research Institute, Giza, Egypt. These were pregnant females rats of 13 weeks old and average weight of $235 \pm 10 \mathrm{~g}$. These have been employed at the end of pregnancy that have been accomplished in proper time according to UFAW $\left(2000{ }^{(8)}\right)$. Animal groups $(6 \times 5=30$ animals) were fed on basal diet for few 
days for adaptation according to Bowman

et al, (1990)(9), meanwhile salt and vitamins mixtures have followed that of AOAC (1984 $\left.{ }^{(10)}\right)$. These pregnant females detected at almost that same time of delivery were divided into five groups and fed the diets outlined in the Table 1 . The food and water were daily given ad libitum. Each mother was kept with its offspring of equal numbers in a separate cage.

To test the biological effect of these food formulations, rats' blood samples were taken and animals were anathesized. The organ were separated and weighed when the trial has been terminated.

Analytical Methods: The triglycerides: (TG) were determined by an enzymatic methods described by Lowell et al., (1973)(11). Total cholesterol (TC) were determined according to the method of Allain et al., (1974)(12). The blood glucose level $(\mathrm{G})$ has been measured according to Dacie and Lowis (1984)(13).

The determination of liver function: The
GPT and GOT in serum were tested by the method described by Reithman and Frankel (1957) $)^{(14)}$.

For cell count, Excel 80 blood cell counter (Danam, USA) was used to count the RBC and WBC, red and weight blood cells, and their fractions.

Histopathological examination of liver: The liver of each mother was collected and post-mortal examination was done as soon as possible. Fixation was carried out in $10 \%$ of natural formalin, dehydrated, cleared, and ended paraffin then sectioned at (4-6 mm), and stained with harris hematoxylin, and casein for histopathological examination (Frankel and Reithman, 1963)(15).

A value of average of 6 sample was calculated to be recorded.

\section{RESULTS AND DISCUSSUN}

In the current study on lactation, dates (D) in diet was examined in supporting this physiological needs in rats. The other food components used, with either of 25 or $50 \%$ 
D, were the sckimmilk (SM) of 20 or $10 \%$ plus another source for $\mathrm{CHO}$ such as sweet potatoes (SP) at a variable ratio of 10,35 , or $25 \%$, respectively. Table (1) shows also the composition of control (C) as well as levels of starch (S), vitamins (VM), plus a special specie mixture of extract as additives (AD) and fat of $3 \%$ used in these groups. The ratio of proteins in diet, almost $17.5 \%$, was corrected using 20 or $10 \%$ of a plant-rich protein antioxidant semimodified food (AC) as seen in Table (1).

Table 1: Groups of date's formulations.

\begin{tabular}{cccccccccc}
\hline Group & $\boldsymbol{D}$ & $\boldsymbol{A C}$ & $\mathbf{S M}$ & $\boldsymbol{S P}$ & $\boldsymbol{S}$ & $\boldsymbol{V} \boldsymbol{M}$ & $\boldsymbol{M} \boldsymbol{M}$ & $\boldsymbol{A D}$ & $\boldsymbol{F a t}$ \\
C & - & - & 46 & - & 46 & 1 & 4 & - & 3 \\
G1 & 50 & 20 & 10 & 10 & - & 1 & 4 & 2 & 3 \\
G2 & 50 & 10 & 30 & - & - & 1 & 4 & 2 & 3 \\
G3 & 25 & 20 & 10 & 35 & - & 1 & 4 & 2 & 3 \\
G4 & 25 & 10 & 30 & 25 & - & 1 & 4 & 2 & 3 \\
\hline
\end{tabular}

Where: $D$ date, AC protein antioxidant semimodified food, SM skim milk, SP sweet potato, $S$ starch, VM and MM are vitamin and mineral mixtures. $A D$ is a mixture of special extract.

As recorded in Table (2), all groups forth week. However, rapid weight loss made a remarkable loss of body weight through diet or medication should not be (BW) during the first week of lactation, attempted during lactation (Bayol, 2007(1), especially the NC assuming a temporary case of malnutrition. The G4, and at a lesser extent G1, were an exception making almost less than 5\% BW reduction during the third week and recover in the and the BW recover is most probably due to what so called the hyperphagia. The BW lose should be prevented by more food amount and quality for both pregnant and lactation must be arranged. It has been found 
Table 2: Mother's body weight changes in four weeks after delivery according to groups of date's formulations.

\begin{tabular}{cccccc}
\hline Groups & BD & $1^{\text {st }}$ wk & $2^{\text {nd }} w k$ & $3^{\text {rd }} w k$ & $4^{\text {th }} w k$ \\
C & $235+/-10$ & $206+/-15$ & 174 & 187 & 180 \\
G1 & $235+/-10$ & $206+/-15$ & 181 & 190 & 200 \\
G2 & $235+/-10$ & $206+/-15$ & 182 & 173 & 180 \\
G3 & $235+/-10$ & $206+/-15$ & 186 & 186 & 195 \\
G4 & $235+/-10$ & $206+/-15$ & 205 & 195 & 200 \\
\hline
\end{tabular}

Means of 6 animals. BD before delivery.

that food intake increased by 10\%, meanwhile, G3 and G4 lose only approximately $60 \%$ during pregnancy and $2.5 \%$ of their BW. It can be concluded that a further $250 \%$ during lactation (Cripps and both $\mathrm{G} 1$ and $\mathrm{G} 4$ are the two diets that may Williams 1975)(2). Moreover, and during lactation, the alimentary canal the entire period of lactation

progressively increased in weight and size (Cripps and Williams, 1975)(2), and this is an adapted hormonal shift (HS) that conjugated this temporary physiological status. It seems that SM and SP, at low date level, or even AC, at high date level, have a particular role in preserving lactating HS including body weight during this period. In some other words, AC posses a singular positive effect at higher level of dates. Again, it is such a hormonal program that connected to feeding category. In this regard, however, the $\mathrm{C}$ and $\mathrm{G} 2$ did make a reduction more than
The offspring growth data, as in Table (3) where the mother milk was their lonely source of feeding, shows that the most growth is accomplished during the last two weeks of lactation. Noticed that the timing is parallel. However, it has been found also that human hypothalamic NPY mRNA was unchanged in pregnancy, moderately increased after 5 days lactation of control that normally fed and increased further at 14 days lactation with semistarvation (Wilding, et al 2002)(3). Likewise, the absolute absorption for leucine and of glucose was increased during lactation, but 
greatest absorption occurred on the 10th day of lactation. Results for absorption of leucine and glucose per unit length indicated that the ability of the mucosal cells to absorb or the number of absorptive cells $/ \mathrm{mm}$ had changed during lactation and the post-lactation periods (Cripps and Williams, 1975)(2). This should be met with more dietary proteins (Zambrano, et al, 2005))(16).

Table 3: Infant (8+/-1) and their body and total body weight during and after four weeks of delivery according to groups of date's formulations.

\begin{tabular}{ccccccc}
\hline Group & \multicolumn{7}{c}{ Infant starting main } & body weight and in four weeks after delivery \\
& MW & 0 & 1 & 2 & 3 & 4 \\
C & 91 & 5.1 & 8.2 & 10 & 13 & 13 \\
G1 & 144 & 5.4 & 9.9 & 12 & 17 & 18 \\
G2 & 182 & 5.2 & 11.5 & 18 & 20 & 26 \\
G3 & 162 & 5.4 & 8.4 & 11 & 17 & 18 \\
G4 & 200 & 5.0 & 9.7 & 13 & 18 & 25 \\
\hline
\end{tabular}

This may explain the drop in BW upon delivery and the beginning of lactation, concerning energy expenditure, then the high rate of recover potential appeared afterwards. Although the $\mathrm{C}$ group showed very poor recovery, G4 was seen to be the most recoverable one. Accordingly, this diet seemed to be the best of them both for mother health and lactation process. However, it has been found that additional servings from the fruit, vegetable, bread, and cereal groups is necessary (Bayol,
2007)(1). Moreover, water, milk and fruit juices are excellent choices for the new mother. In particularly, Zambrano, et al. $(2006)^{(17)}$ stated that low maternal protein diet during pregnancy and lactation has sex- and window of exposure-specific effects on offspring growth and food intake, glucose metabolism and serum leptin in the rat. It is supposed to minimize the amount of empty calorie foods consumed, so that there is more appetite for nutrient rich foods. In some other wards, G4 diet seems 
to be rich as balanced food of variety, Table (4). It is noticed that C is little bit over hence no one food or food group should be in the whole mentioned blood parameter. eliminated from the diet $(B a y o l, 2007)^{(1)}$. Much more TC, TG and G elevations were

In biological evaluation for the dietary recorded in both1 and 2 groups. Similar pattern used during lactation, blood trend is shown for liver enzymes. analysis and liver function are listed in

Table 4: Mother's blood analysis and liver enzyme diagnostic according to groups of date's formulations.

\begin{tabular}{ccccccc}
\hline Groups & TG & TC & Glucose & AST & ALT & Ranking \# \\
\hline C & $112.6 / 100$ & $89.3 / 100$ & $88.5 / 100$ & $396.2 / 100$ & $89.4 / 100$ & 1 \\
G1 & $141.1 / 125$ & $116.8 / 132$ & $97.5 / 110$ & $446.2 / 113$ & $121.3 / 136$ & 3 \\
G2 & $133.2 / 118$ & $121.2 / 136$ & $91.2 / 103$ & $405.3 / 102$ & $119.4 / 134$ & 2 \\
G3 & $102.6 / 91$ & $98.6 / 111$ & $74.3 / 84$ & $366.3 / 92$ & $69.8 / 79$ & 1 \\
G4 & $100.1 / 89$ & $69.8 / 79$ & $71.3 / 81$ & $302.2 / 76$ & $63.7 / 72$ & 1 \\
\hline
\end{tabular}

$\mathrm{Mg} / \mathrm{dl}$ or U/mI serum / percent NC

Better blood and liver function are obtained for the other groups especially G4. In biological evaluation using blood cells count of mothers, as in Table (5), data concluded that G4 system of dieting exhibited the highest WBC plus the greatest cell fractions in addition to the most countable two fragments in RBC, and predisposed that G3 seems to be the most acceptable blood cell counts afterwards.

Organ in lactating rats under the condition of the present experiment doesn't show any particular shape, i.e., the largest, within normal range: liver, spleen, lung, kidney, and heart were recorded for G4, G1, NC, NC, G3 or G4, G3, G4 and G3 in the same respect. The changing in organ size and hormonal balance with lactation is true. Pancreas weight, for instance, was increased $41 \%$ in a similar rat experiment as well as contents of DNA, RNA, and protein (McLaughlin, et al, 1983)(6). Thus, 
hyperphagia in lactating female rats was associated with decreased sensitivity to the satiety effect of CCK similar to that in hyperphagic obese rats and hypertrophy of the pancreas in contrast to decreased pancreas size in obese rats (McLaughlin, et al, 1983) ${ }^{(6)}$.

More remarkable substantial differences were noticed in tissues. Tables (7 and 8) illustrated that there is somewhat similar effect for diet under investigation on liver and kidney histopathology.
This patterns of biological evaluation reflect the greatest absorption occurred in subsequent days of lactation that go in harmony with the present data. The ability of the mucosal cells to absorb or the number of absorptive cells $/ \mathrm{mm}$ is changed during lactation and the post-lactation periods (Cripps and Williams, 1975)(2).

Despite the some minor defect, the integral achievement of the biological tools used besides the mothers and infant appropriate total health is giving the priority to dietary system of group 4 .

Table 5: Mother's blood cell counts according to groups of date's formulations.

\begin{tabular}{cccccccccc}
\hline Group & WBC & RBC & HGB & HCT & LYM & MONO & ESO & BASO & LUC \\
\hline C & 5.04 & 6.38 & 12.6 & 39.9 & 33.4 & 21.5 & 2.3 & 0.4 & 5.7 \\
G1 & 3.46 & 6.02 & 12.0 & 36.5 & 52.0 & 24.6 & 2.5 & 0.1 & 6.9 \\
G2 & 3.46 & 5.74 & 12.7 & 36.6 & 47.3 & 20.4 & 7.1 & 0.9 & 3.6 \\
G3 & 5.04 & 6.74 & 13.1 & 41.5 & 67.1 & 11.3 & 0.9 & 0.1 & 7.8 \\
G4 & 8.30 & 5.84 & 14.4 & 47.4 & 44.7 & 12.7 & 1.1 & 0.1 & 8.3 \\
\hline
\end{tabular}

Excel 80 blood cell counter (Danam, USA). RBC and WBC are red and weight blood cells and their fraction.

Table 6: Mother's organ weight changes according to the food formulations.

\begin{tabular}{cccccc}
\hline Group & Liver & Spleen & Lung & Kidney & Heart \\
\hline NC & 5.40 & 0.70 & 1.80 & 1.70 & 1.00 \\
G1 & 6.30 & 1.40 & 1.70 & 1.70 & 1.00 \\
G2 & 5.75 & 1.35 & 1.30 & 1.40 & 1.15 \\
G3 & 6.30 & 1.20 & 1.20 & 1.40 & 0.70 \\
G4 & 7.30 & 1.20 & 1.23 & 1.30 & 1.00 \\
\hline
\end{tabular}


Table 7: Effect of feeding rat's date's formulations on liver histopathology.

\begin{tabular}{|c|c|c|c|c|c|}
\hline Groups & NC & G1 & G2 & G3 & $\overline{\text { G4 }}$ \\
\hline Hepatomegaly, karyomegaly and a typical mitosis & - & + & - & - & - \\
\hline Hepatic sinusoids of sporadic hepatocyte & - & + & - & - & - \\
\hline Oval cells proliferation & - & + & - & - & - \\
\hline $\begin{array}{l}\text { Hyperplasia, activation and proliferation of epithelial } \\
\text { lining }\end{array}$ & - & + & - & - & - \\
\hline \multicolumn{6}{|l|}{$\begin{array}{l}\text { Bile duct associated with formation of newly formed } \\
\text { bileDuctless. }\end{array}$} \\
\hline Leucocytic cells infiltration in the portal triodes & - & $2+$ & + & - & - \\
\hline $\begin{array}{l}\text { Kypffer cells activation and hydropic degeneration of } \\
\text { hepatocytes }\end{array}$ & - & - & $2+$ & - & - \\
\hline Vaculation of cytoplasm of some hepatocytes & - & - & - & + & - \\
\hline necrosis sporadic & - & - & - & - & $+/-$ \\
\hline Ranking \# & 1 & 3 & 2 & 1 & 1 \\
\hline
\end{tabular}

Table 8: Effect of feeding rat date's formulations on kidney histopathology.

\begin{tabular}{lllllll}
\hline \multicolumn{1}{c}{ Groups } & NC & 1 & 2 & 3 & 4 \\
\hline $\begin{array}{l}\text { Renal tabules vaculation of epithelial lining } \\
\text { Focal area of tubular necrosis associated }\end{array}$ & - & + & - & - & - \\
leucocytic Cells infiltration & - & + & - & - & + \\
$\begin{array}{l}\text { Hypertrophy of glomerular tufts and vaculation of } \\
\text { Endothelial lining glomerular tafts }\end{array}$ & - & + & - & - & - \\
$\begin{array}{l}\text { Thickening of the glomerrular basement membrane } \\
\text { and Partial layer of Bowman's capsule }\end{array}$ & - & + & - & - & - \\
$\begin{array}{l}\text { Atrophy of some glomerular tufts } \\
\text { Apparent normal renal parenchyma }\end{array}$ & - & & & & \\
$\begin{array}{l}\text { Presence of esonophilic protienaceous cast in lumen } \\
\text { of Some tubules. }\end{array}$ & - & - & - & $-/+$ & - & - \\
Necrobiotic of epithelial lining renal tubules & - & - & - & + \\
Ranking \# & - & - & - & - & + \\
\hline
\end{tabular}

In conclusion, the improvement of blood picture as well as both immune cells and $\mathrm{RBC}$ among its cell fragments besides the microscopic appearance of tissues may be considered a good biological indication for the appropriate balanced dietary system used, i.e., G4. This system most probably met the new existed hormonal situation and matches the appropriate physiological requirements. Notice the similarity in ranking number both in brain and liver.

Dietary therapy including diet effect on hormonal balance is clear in this special physiologic case. Here, for hormonal 
adaptation, neuropeptide $\mathrm{Y}$ is the most powerful appetite stimulant known, and rates of synthesis and release in the hypothalamus correlate closely with nutritional status (Wilding, et al. 2002)(3). Increases in hypothalamic NPY synthesis may partly be responsible for the increase in food intake seen in lactation, but unlike in food deprivation, the increase is not related to circulating insulin, suggesting involvement of other regulatory factors ${ }^{(3)}$. It might be important to mention that perturbed skeletal muscle insulin signaling in the adult female intrauterine growthrestricted rat is a hormonal interaction (Oak et al. 2006)(18). Further studies, therefore, will be conducted in this area.

\section{REFERANCES}

1- Bayol SM. A maternal 'junk food' diet in pregnancy and lactation promotes an xacerbated taste for 'junk food' and a greater propensity for obesity in rat offspring, Welcome Trust. British Journal of Nutrition. published online 2007.

2- Cripps AW, Williams VJ. British $\mathrm{J}$ of Nutr, Volume 33, Number 1, January 1975.
3- Wilding JPH, Ajala MO, Lambert PD, Bloom SR. Additive effects of lactation and food restriction to increase hypothalamic NPY mRNA in rats. Journal of Endocrinology. 2002;152: 3.

4- Williams RD. Breast-Feeding Best Bet for Babies. FDA Consumer Magazine. 1995.

5- Douglas AJ, Johnstonea LE, Lenga G. Neuroendocrine mechanisms of change in food intake during pregnancy: A potential role for brain oxytocin. Physiology \& Behavior. Elsevier Inc; 2007.

6- McLaughlin CL, Baile CA, Peikin SR. Hyperphagia during lactation: satiety response to CCK and growth of the pancreas. Am J Physiol Endocrinol Metab. 1983; 244: E61-E65.

7- Ahmed AIS, Habib GM, Saad SAM, El-Deeb F. Relation of natural antioxidants to hormonal balance and immune system. Egy J Biomed Sci, 2005;17, 294-305.

8- UFAW. Handbook on the care and management of laboratory Animals Seventh edition. 2000; vol.1

9- Bowman TA, Gonewardene M, Taylor CE. VA deficiency decrease NKC activity. J Nutr. 1990;120,1264-73.

10- AOAC. Official Methods of Analysis. $14^{\text {th }}$ ed. USA: AOAC;1984.

11- Lowell R, Rosebrough A, Fan J, Randall A. A simplification of protein assay method of Lower et al. Ann Biochem. 1973;83:346.

12- Allian CC, Poon LS, Chan CS. Enzymatic determination of total serum cholesterol. Clin. Chem.1974; 20:470-5.

13- Dacie JV, Lewis SM. Practical hematology. New York: Churchill Livingston; 1984.

14- Reithman S, Frankel S. A calorimetric method for the determination of serum glutamic oxalacetic and glutamic 
pyrovic transaminase" Am J Clin Path. 1957;28,56.

15- Frankel S, Reitman S. Clinical laboratory methods" USA: The C.V. Mosbye Company; 1963.

16- Zambrano EP, M Martinez-Samayoa M, Bautista CJ, Deas M, L Guillen G, Rodriguez-Gonzalez L, et al. Sex differences in transgenerational alterations of growth and metabolism in progeny (F2) of female offspring (F1) of rats fed a low protein diet during pregnancy and lactation. J Physiol. 2005; 566(1): 225 - 36.

17- Zambrano EM, Bautista CJ, Deas M, Martinez-Samayoa PM, M. Gonzalez-
Zamorano $\mathrm{M}$, Ledesma $\mathrm{H}$, et al. A low maternal protein diet during pregnancy and lactation has sex- and window of exposure-specific effects on offspring growth and food intake, glucose metabolism and serum leptin in the rat. J Physiol. 2006; 571(1): 221 - 30.

18- Oak S A, Tran C, Pan G, Thamotharan $M$, Devaskar SU. Perturbed skeletal muscle insulin signaling in the adult female intrauterine growth-restricted rat. Am J Physiol Endocrinol Metab. 2006; 290(6): E1321 - 30. 